\title{
Orbital determination and dynamics of resonant extrasolar planetary systems
}

\author{
C. Beaugé ${ }^{1}$, S. Ferraz-Mello ${ }^{2}$, T. A. Michtchenko ${ }^{2}$ \\ and C. A. Giuppone ${ }^{1}$ \\ ${ }^{1}$ Observatorio Astronómico, Universidad Nacional de Córdoba, Laprida 854, (X5000BGR) \\ Córdoba, Argentina \\ ${ }^{2}$ Instituto de Astronomia, Geofísica e Ciências Atmosféricas, USP, Rua do Matão 1226, \\ 05508-900 São Paulo, Brasil
}

\begin{abstract}
In this communication we review some properties and applications of mean-motion resonances in extrasolar planetary systems, with particular emphasis on the $2 / 1$ commensurability. A first part is devoted to the dynamical structure of the $2 / 1$ resonance, including (but not restricted to) the so-called apsidal corotations. In a second part we discuss the orbital evolution of resonant systems under the effects of non-conservative forces. Special attention is given to the use of apsidal corotations as markers of largescale orbital decay, possibly due to disk-planet interactions in primordial times. Finally, we analyze the interplay between dynamical analysis and orbital fitting. Using the HD82943 planetary system as an example, we discuss: (i) up to what point present orbital fits allow us to distinguish between different resonant configurations, and (ii) in what ways may the dynamical structure of resonances be used as a complementary part of the orbital fitting process.
\end{abstract}

Keywords. celestial mechanics, planetary systems, planets and satellites: general

\section{Introduction}

A mean-motion resonance (MMR) occurs when the ratio of their orbital periods lies close to the ratio of two small integers. We can write this condition in a generic form as

$$
\frac{n_{1}}{n_{2}} \simeq \frac{(p+q)}{p}
$$

where $n_{i}$ are the mean motions (orbital frequencies) of the planets and $p, q$ are integers. Following general usage, we identify the planet with smaller semimajor axis by the index 1 , while 2 is reserved for its outer companion (i.e. $a_{1}<a_{2}$ ). In the planar case, the dynamics of a resonant system is characterized by the evolution of two critical (or resonant) angular variables, defined as

$$
\theta_{i}=(p+q) \lambda_{2}-p \lambda_{1}-q \varpi_{i} \quad(i=1,2),
$$

where $\lambda_{i}$ are the mean longitudes and $\varpi_{i}$ the longitudes of pericenter. The value of $q$ defines the order of the resonance.

If at least one of the critical angles is found to be librating, then the system is said to be inside the mean-motion resonance. For the present work, we define libration as the case in which the angle does not take all values between zero and $2 \pi$, but oscillates with a certain amplitude around an equilibrium value $\theta_{i_{0}}$. We will not analyze whether this libration is dynamical (i.e. associated to a separatrix crossing) or merely kinematical, but concentrate solely on the observed behavior of the variable under question. If neither angular variable librates, the system is said to be outside the resonance, or in a nonresonant configuration. 
Definition (1.2) implies that $\theta_{1}-\theta_{2}=q\left(\varpi_{2}-\varpi_{1}\right)=q \Delta \varpi$, with $\Delta \varpi$ the difference in the longitudes of the pericenter. This a a purely secular (non-resonant) angle and its behavior defines the secular evolution of the system. Analogous to a MMR, if $\Delta \varpi$ librates, the system is said to be in an apsidal alignment. Usually this behavior is referred to as a secular resonance, although Michtchenko and Malhotra (2004) showed that true secular resonance -dynamical libration- only occur for very high eccentricities (typically of the order of 0.7). Thus, for most cases the apsidal alignment is a purely kinematical phenomenon, and no separatrix divides the librational and circulatory regions.

Since rational numbers form a dense set in the real axis, equation (1.1) does not appear to be a very strict condition. At least in principle, any two planets (with arbitrary semimajor axes) could be said to be resonant, provided the values of $p, q$ are chosen sufficiently large. However, it can be shown (e.g. Murray and Dermott 1999) that, unless the values of $p, q$ are small integers, the magnitude of the resonant terms in the perturbation are smaller than the short period contributions, and the corresponding resonance is not effective in dominating the dynamics of the system. Thus, only small order resonances are important: $2 / 1,3 / 2,3 / 1,5 / 3$, etc. Of these, the strongest is the $2 / 1$, corresponding to the smallest integers $(p=q=1)$.

Since the important resonances only occupy a very limited region in the domain of semimajor axis, it is not expected that planetary systems should be largely associated to MMR. However, this does not appear to be the case. Among the 26 presently known multiple-planetary systems, 13 contain planets in nearby orbits in which their mutual perturbations are significant. Of these, probably 8 are in the immediate vicinity of meanmotion resonances. Of course, many of them have been detected only recently, and their orbits are still not well determined. Consequently, it is not certain whether all seemingly resonant configurations are real. Nevertheless, the proportion of resonant systems is still very significant.

Among the different resonant systems, most of them seem to be associated to the $2 / 1$ MMR. These are GJ876, HD82943, HD73526, HD128311 and HD160691. Two planets in $55 \mathrm{Cnc}$ are believed to lie within the $3 / 1 \mathrm{MMR}$, although the most recent orbital fit by Fischer et al. (2007) seems to place these planets outside MMR. Other examples could also exist in higher-order commensurabilities, such as HD202206 and HD12661 in the 5/1. However, these resonances are very weak, and the statement of their resonant motion is more related to stability criteria than to observational evidence. Even so, it appears that approximately $8 / 13(\approx 61 \%)$ of the presently known planetary systems in nearby orbits contain bodies associated to MMR. Even if some are questionable and are later removed from the list, the percentage is still very significant, indicating that the frequency of resonant systems among extrasolar planets is much larger than expected from a random distribution of orbits.

In our own Solar System, a similar picture occurs in satellite systems of the outer planets, where many of the regular moons display resonant motion. Probably the best known example are the three inner Galilean satellites of Jupiter (Io, Europa and Ganymedes). Not only are these bodies locked in two successive 2/1 MMR, as the orbital configuration of all three lie in the so-called Laplace resonance where the resonant angle $\theta_{L}=\lambda_{E}-3 \lambda_{G}+2 \lambda_{I}$ librates around zero with an amplitude of less than one tenth of a degree. As far back as fifty years ago (e.g. Roy and Ovenden 1954) it was known that the frequency of resonances in the satellite systems is much higher than expected. It was later shown that these configurations could be explained by orbital evolution due to tidal effects (Goldreich 1965). These resonances are not primordial, but a consequence of resonance trapping due to an exterior non-conservative force.

In the past years, resonance capture has also been proposed for extrasolar planets, although in this case the driving mechanism is not tidal friction but interactions between 
the planets and planetesimals or gaseous disks. However, a good scenario must not only explain the presence of planets in resonance, but also the specific resonant configurations in which they presently lie. For that sake, we must understand the structure of the phase space associated to planetary mean-motion resonances, identify possible regions of motion for planetary bodies, and analyze which solutions are compatible with planetary migration.

\section{Dynamics of Planetary Mean-Motion Resonances}

In the vicinity of a MMR, limiting ourselves to coplanar orbits, the gravitational potential of the interaction between both planets (the so-called disturbing function) can be written in a generic form as

$$
\mathcal{R}=\sum_{j_{1}, j_{2}, j_{3}} A_{j_{1}, j_{2}, j_{3}}\left(a_{1}, a_{2}, e_{1}, e_{2}\right) \cos \left(j_{1} Q+j_{2} \theta_{1}+j_{3} \Delta \varpi\right)
$$

(e.g. Beaugé and Michtchenko 2003) where the index $j_{i}$ can take any integer value, $A_{j_{1}, j_{2}, j_{3}}$ are coefficients that depend on the semimajor axes and eccentricities, and $Q=$ $\lambda_{1}-\lambda_{2}$ is the synodic angle. Perturbation terms with $j_{1}=j_{2}=0$ are usually called secular terms. Terms that only depend on $\theta_{1}$ and $\Delta \varpi$ (i.e. $j_{1}=0$ ) are called resonant perturbations, while those that contain $Q$ in their arguments are usually known as short-period terms. If the resonance relation is dominant, short-period contributions have smaller amplitudes (i.e. small $A_{j_{1}, j_{2}, j_{3}}$ ) and their effects cancel out over longer timescales. Thus, if we are interested in the long-term dynamics of the system, we can eliminate the gravitational interactions associated to the angle $Q$ and reduce the disturbing function to resonant and secular terms only. This averaging of $\mathcal{R}$ can be carried either by analytical (e.g. Beaugé and Michtchenko 2003) or semi-analytical methods (e.g. Michtchenko et al. 2006).

The averaged resonant planetary problem (planar case) then depends only on two independent angles $\theta_{1}$ and $\Delta \varpi$, and constitutes a two degrees-of-freedom dynamical system. If the orbital elements are not astrocentric, but calculated with Jacobi or Poincaré canonical variables, then the problem contains three integrals of motion, given, up to second order in the masses, by:

$$
\begin{aligned}
& \mathcal{F}=G \frac{m_{0} m_{1}}{2 a_{1}}+G \frac{m_{0} m_{2}}{2 a_{2}}-\frac{1}{2 \pi} \int_{0}^{2 \pi} \mathcal{R} d Q \\
& \mathcal{L}=m_{1} n_{1} a_{1}^{2} \sqrt{1-e_{1}^{2}}+m_{2} n_{2} a_{2}^{2} \sqrt{1-e_{2}^{2}} \\
& \mathcal{K}=(p+q) m_{1} n_{1} a_{1}^{2}+p m_{2} n_{2} a_{2}^{2}
\end{aligned}
$$

where $\mathcal{F}$ is the Hamiltonian (total orbital energy), $\mathcal{L}$ is the total angular momentum and $\mathcal{K}$ is sometimes referred to as the spacing parameter (see Michtchenko and Ferraz-Mello 2001, Michtchenko et al. 2008). Given any initial condition in the immediate vicinity of the MMR, its orbital evolution will be such that all three functions remain constant for all time. It is important to emphasize that expressions (2.2) are not integrals of motion if the orbital elements are astrocentric.

Equilibrium solutions constitute a particular, but very important, type of configurations. They are given by the conditions

$$
\frac{d a_{1}}{d t}=\frac{d a_{2}}{d t}=\frac{d e_{1}}{d t}=\frac{d e_{2}}{d t}=\frac{d \theta_{1}}{d t}=\frac{d \Delta \varpi}{d t}=0
$$

and correspond to periodic orbits in the unaveraged system. If the solution is stable, 


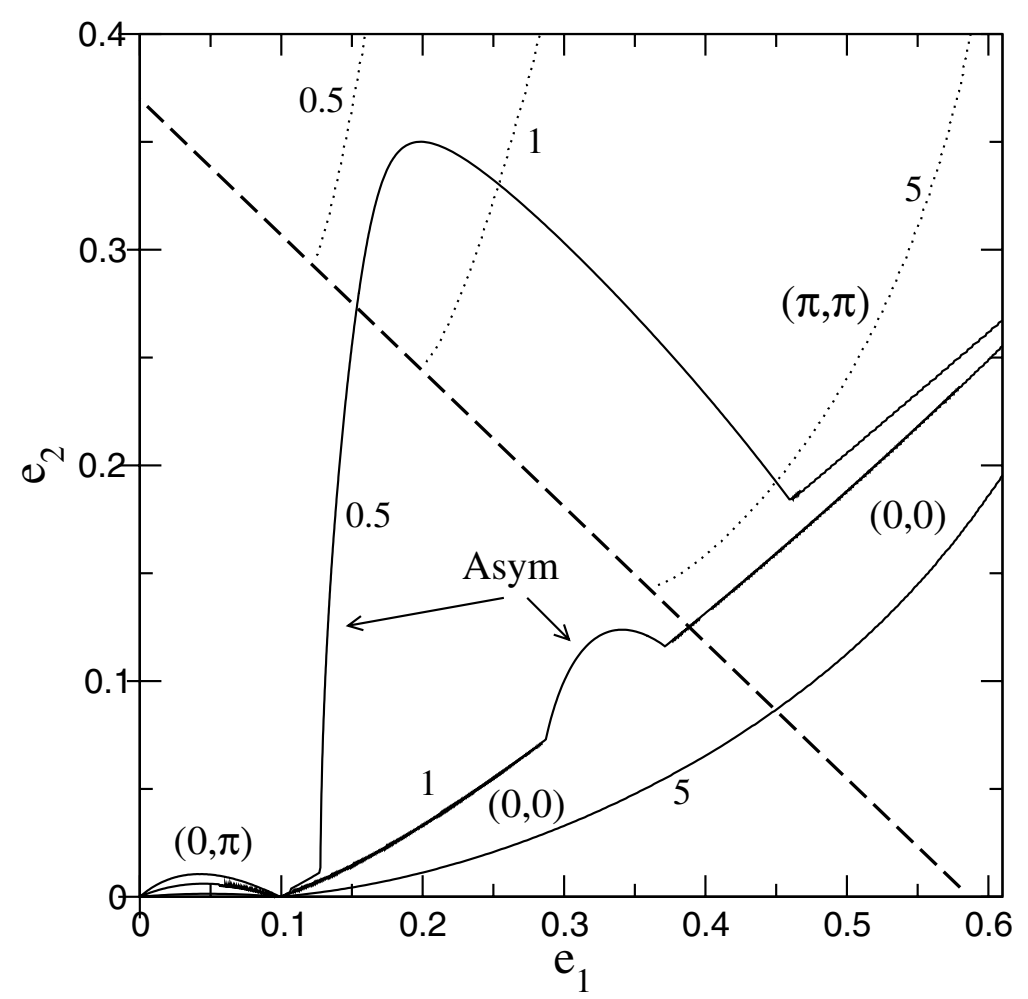

Figure 1. Families of stable zero-amplitude ACR for the $2 / 1 \mathrm{MMR}$, in the plane of the eccentricities, and for three different mass ratios $m_{2} / m_{1}=0.5,1,5 .(\pi, \pi)$-ACR (dotted lines) exist only above the collision curve, shown as a dashed diagonal line. Other ACRs are shown in continuous curves.

initial conditions in its vicinity will display oscillations around the stationary point. In particular, both the resonant angle $\theta_{1}$ and the secular angle $\Delta \varpi$ will librate around fixed values. Such behavior corresponds to a simultaneous capture into a mean-motion and a secular resonance; we have called such solutions Apsidal Corotation Resonances, or ACR for short (Ferraz-Mello et al. 2005b). Among the numerous families of ACR (See Michtchenko et al. 2006 for a catalog of ACR for several MMR), the most important are those associated to global maxima of the Hamiltonian function $\mathcal{F}$. From (2.2) and (2.3) it can be seen that maxima of $\mathcal{F}$ correspond to minima of the disturbing function $\mathcal{R}$. In other words, these ACR are configurations where the gravitational interactions between the planets are minima, and are thus preferential stable configurations for resonant planets. In the case of high-eccentricity planetary systems, where physical encounters are possible and most orbital configurations invariably lead to unstable motion, ACR is an effective mechanism that protects planets from close approaches and allow the system to survive.

The protective role of ACR makes these solutions natural nesting places for moderateto-high eccentricity planetary systems, and it is not surprising that many (if not all) resonant planets have stable orbital fits consistent with ACR. Perhaps the most wellknown example is GJ876 (e.g. Laughling and Chambers 2001, Lee and Peale 2002), where both outer planets are locked in an ACR inside the 2/1 MMR, displaying small amplitude librations around $\theta_{1}=\Delta \varpi=0$. We refer to such a solution as a $(0,0)$-ACR, where the numbers in the brackets mark the equilibrium value of $\theta_{1}$ and $\Delta \varpi$, respectively. HD82943 is another example. Up to 2003, the two planets of this system were believed 
to be locked in a $(\pi, \pi)$-ACR (oscillations around $\theta_{1}=\Delta \varpi=\pi$ ), although later updates in the masses and orbital elements placed the system in an unstable orbit around a $(0,0)$-ACR. Finally, until recently the orbital fit of the planets $55 \mathrm{Cnc}-\mathrm{c}$ and $55 \mathrm{Cnc}-\mathrm{d}$ was consistent with an ACR in the $3 / 1 \mathrm{MMR}$, where both $\theta_{1}$ and $\Delta \varpi$ librate around values different from 0 or $\pi$. We call this solution an asymmetric-ACR (Beaugé et al. 2003, Ferraz-Mello et al. 2003). With the discovery of the fifth planet in 55Cnc (Fischer et al. 2007), the new orbital solution puts the planets outside the $3 / 1$ commensurability. However, as we will discuss in next section, orbital fits for multi-planetary systems are very imprecise and often show solutions with different dynamical behavior within the 1- $\sigma$ level of confidence of the best fit. Thus, it is perhaps too early to discard the resonant relation for this system, at least until further observations confirm the more recent fits.

In Beaugé et al. (2003) we showed that, at least in a first approximation, the location of the ACR depends on the planetary masses and semimajor axes only through the ratios $m_{2} / m_{1}$ and $a_{1} / a_{2}$. This permits a global study of ACR, constructing maps or catalogs of these stationary solutions that will be valid for any planetary system. Figure 1 plots the most important families of stable ACR for the 2/1 MMR in the eccentricities plane, and for three mass ratios (values shown near each curve). Except for $(\pi, \pi)$-ACR that only exist above the collision curve, all the other solutions form continuous families (one per mass ratio) that include quasi-circular orbits. Asymmetric solutions only exist for $m_{2} / m_{1} \leqslant 1.015$ (Ferraz-Mello et al. 2003, Lee 2004); for larger mass ratios only symmetric configurations are found.

Notwithstanding their importance, ACR only give limited information on the resonant structure. To obtain a more complete picture and identify other possible configurations, the analysis must be extended beyond the stationary solutions. A study in this direction is presented in Michtchenko et al. (2008). Although the reader is referred to that paper for a more detailed analysis, here we reproduce some of the results.

Figure 2 shows four dynamical maps of the $2 / 1$ MMR, considering a mass ratio slightly larger than unity. Each map was constructed from the analysis of a grid of initial conditions centered around a given ACR. The gray scale shows the spectral number SN (Michtchenko and Ferraz-Mello 2001) resulting from a Fourier analysis of the eccentricity of the inner planet, obtained from a numerical integration over more than $10^{5}$ orbital periods. White strips mark the presence of stable periodic orbits of the averaged problem, while darker tones indicate increasingly chaotic motion. Zones of forbidden motion are shaded in red, and the red circle in each frame is the stable ACR.

As can be seen in the two top plots, the region accessible to the planets is divided into two zones, separated by a chaotic layer. The inner zone, around the ACR, is the region of resonant motion while, outside, the orbits are non-resonant (i.e. secular). This latter region is dominated by the two modes of secular motion (called Modes I and II), corresponding to apsidal alignments around $\Delta \varpi=0$ and $\Delta \varpi=\pi$, respectively. Inside the resonant region there are two main families of periodic orbits, whose intersection coincides with the ACR. The vertical branch is associated to a fixed value of $\theta_{1}$ and large amplitude variation of $\Delta \varpi$. In the horizontal branch the opposite occurs: $\Delta \varpi$ is fixed while $\theta_{1}$ suffers large amplitude oscillations. As can be seen in the plot for $e_{1}=0.1$, other white strips representing additional families of periodic orbits are also present. These are related to secondary resonances where the frequency of libration of $\theta_{1}$ is commensurable with the circulatory frequency of $\Delta \varpi$.

In the frame for $e_{1}=0.2$ the chaotic layer defining the boundaries of the resonant region has grown significantly, pushing the secular regime practically outside the limits of the graph. The strong chaotic zone implies that the secular and resonant regions are virtually isolated, making any transition difficult. In particular, it helps to explain why 

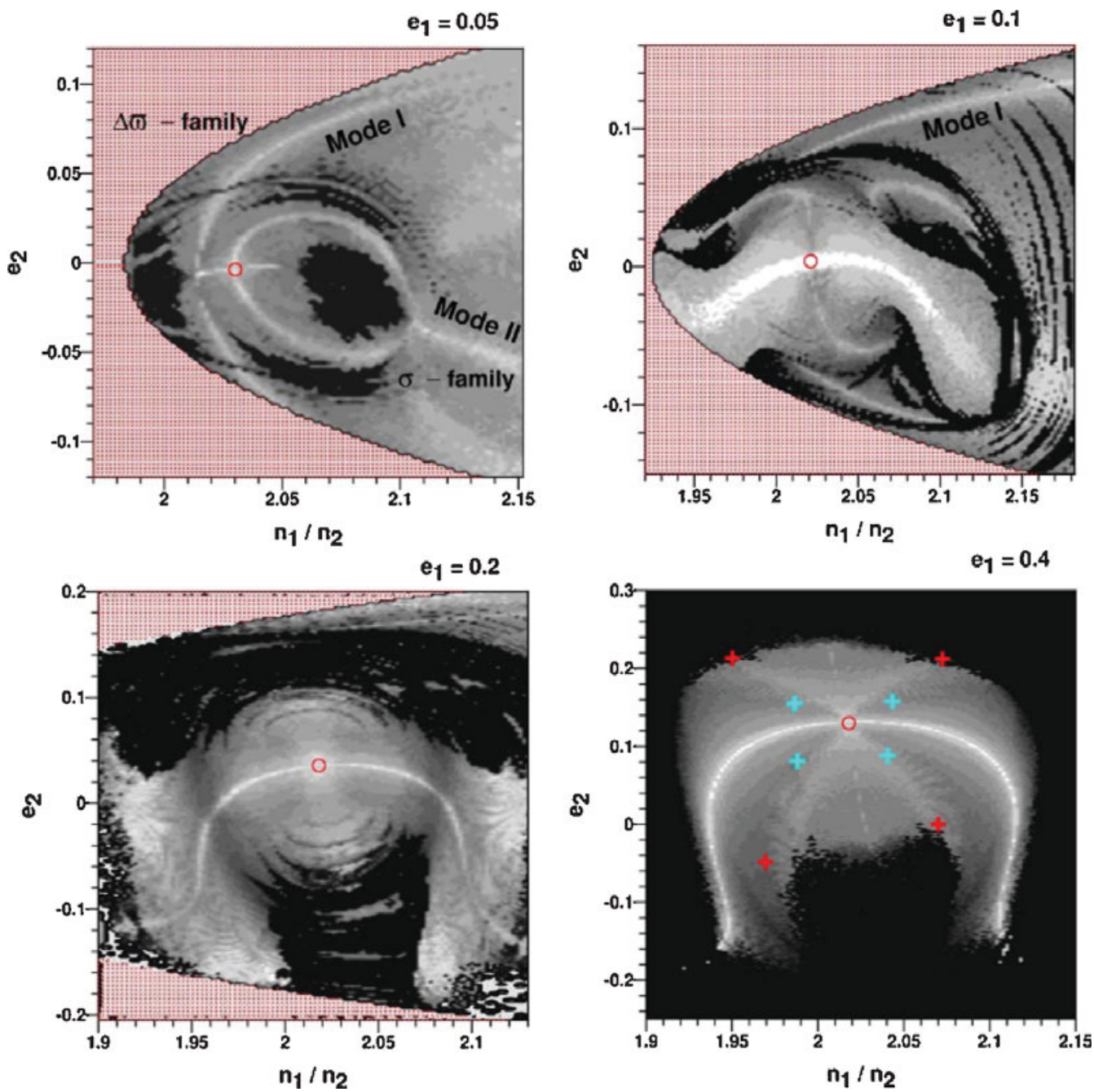

Figure 2. Dynamical maps of the $2 / 1 \mathrm{MMR}$ for $m_{2} / m_{1}=1.064$ around four diffrent symmetric ACR. Each is identified by the eccentricity of the inner planet. Each plot shows the spectral number resulting for a numerical simulation for 130000 orbital periods. Initial conditions were chosen with equal values of the angular momentum integral $\mathcal{L}$ and scale parameter $\mathcal{K}$. In each frame, stable ACR is identified by a red circle, periodic orbits by white strips, and chaotic motion by increasingly dark tones of gray. The red dotted region corresponds to forbidden initial conditions. The indicated colors refer to the electronic version of the paper. Figure reproduced from Michtchenko et al. (2008).

resonance trapping has a lower probability for initial eccentric orbits. Finally, for $e_{1}=0.4$ the only region with stable motion is associated to resonant motion, and all secular orbits are chaotic with very short lifetimes. For $e_{1}$ larger than some limiting value between 0.2 and 0.4 , the only stable orbits possible for planetary systems in the near vicinity of the 2/1 MMR are resonant, and correspond to oscillations around the ACR. An example, the red crosses in the lower right-hand frame correspond to initial conditions equal to the orbital fit of the HD82943 planets by Mayor et al. (2004), whose dynamical instability appears very clearly from its location at the edge of the resonant region. Conversely, the light blue crosses correspond to the initial conditions given by the stable Fit B 
(Ferraz-Mello et al. 2005a) for the same system. This solution is located much closer to the zero-amplitude ACR, and corresponds to a stable ACR with moderate amplitude.

Figure 2 shows a very complex structure for the $2 / 1 \mathrm{MMR}$, even restricted to the planar case. Especially for lower eccentricities, several different modes of stable motion are present which, in principle, could represent possible configurations for real planetary systems. Curiously, the phase space becomes simpler for growing eccentricities, until all possible stable motion becomes dominated by the central ACR.

\section{Planetary Migration and Resonance Capture}

Extrasolar planets usually have smaller semimajor axes and higher eccentricities than expected from classical cosmogonic theories. Either these planets formed by a mechanism different from the one that gave birth to our Solar System, or the exoplanets suffered significant orbital evolution after their formation. This latter proposal is sometimes known as the Hypothesis of Planetary Migration. Although several migration scenarios have been proposed, such as planetary scattering or interactions with a remnant planetesimal disk, the most probable process is a byproduct of the interactions between the planets and its surrounding gaseous disk. This interaction causes an exchange of energy and angular momentum between both components (gas and solid bodies) leading to an infall of the orbital distance of the planets (e.g. Ward 1997).

Even though the orbital evolution of the semimajor axis of the planet under planetdisk interactions is well established, there is still ongoing discussion as to the evolution of the orbital eccentricity. Depending on the relative strength of the Lindblad and corotational resonances with the disk (Goldreich and Sari 2003), the eccentricity can either increase or decrease as the planet falls towards the star. This is a fundamental issue in the hypothesis of planetary migration. On one hand, the existence of solitary giant planets with large eccentricities points towards an eccentricity pumping scenario. On the other hand, largescale orbital decay of resonant planets requires a damping of the same orbital element (Lee and Peale 2002, Beaugé et al. 2006). Of course planetary systems with only one known mass could hide additional bodies, and the observed eccentricities could have been excited by other means, such as planetary scattering (e.g. Ford et al. 2005) or perhaps even jet acceleration of the host star (Namouni and Zhou 2006).

Although planetary migration has several unsolved problems, it is probably our best explanation for the current orbital characteristics of exoplanets. However, in order to consider it more than just an interesting hypothesis, we require some evidence that it really occurred. Multiple-planet systems in mean-motion resonances are specially suited for this task. Not only may they serve as evidence of such a past migration, but what type of resonant solution they inhabit at present may also give us valuable information about details of the orbital decay itself.

Figure 3 shows the result of a numerical simulation of the orbital evolution of two fictitious planets $\left(m_{1}=1 M_{J u p}\right.$ and $\left.m_{2}=0.5 M_{J u p}\right)$ initially placed in circular orbits with $a_{1}=5.4 \mathrm{AU}$ and $a_{2}=9.5 \mathrm{AU}$. Planetary migration was modeled with a Stokestype dissipative force (Beaugé et al. 2006) which affected only the outer body. The drag coefficients were chosen such that $a_{2}$ decayed with an e-folding time of $\tau_{a}=10^{6}$ years, but did not cause any secular change in the eccentricity (i.e. $\tau_{e}=\infty$ ). Initially only the outer body suffers an orbital decay, while the semimajor axis of the inner planet is practically constant. However, once resonance capture occurs in the $2 / 1 \mathrm{MMR}\left(t \sim 10^{5}\right.$ years $)$, the ratio of mean motions becomes locked. Since the dissipative force is still acting on $m_{2}$, the outer planet still falls, but now with a slower rate since it must push the inner body with it. Although both planets still evolve towards smaller orbits, the resonance relation 

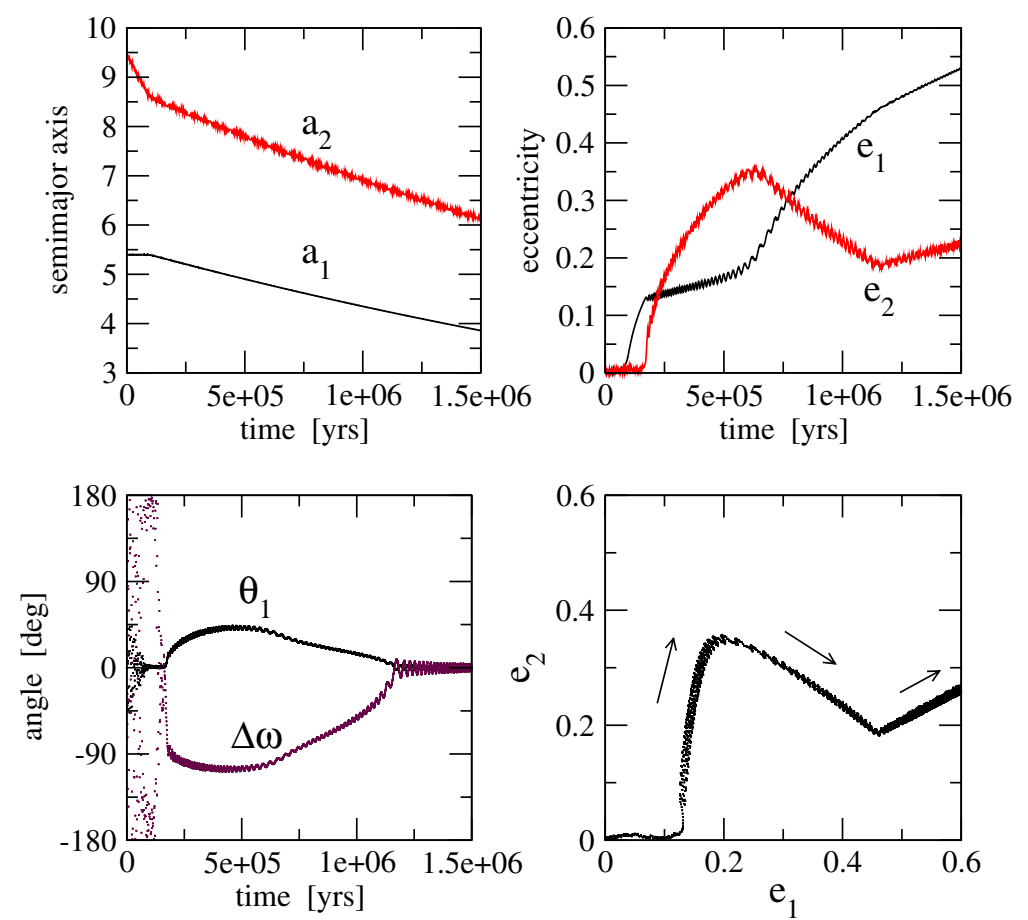

Figure 3. Numerical simulation of planetary migration leading to a capture of two planets $\left(m_{2} / m_{1}=0.5\right)$ in the $2 / 1 \mathrm{MMR}$. Notice the switch from symmetric to asymmetric ACR at $t \approx 2 \times 10^{5}$ years, and back to symmetric at $t \approx 1.2 \times 10^{6}$ years. In the lower right-hand frame, arrows show the direction of orbital variation.

is maintained. As soon as the resonance lock is achieved, the eccentricities begin to grow and the angular variables acquire small oscillations around equilibrium values.

From this point onwards, all orbital evolution occurs within the resonant region of the $2 / 1$ MMR. The lower right-hand frame of Figure 3 shows the relationship between $e_{2}$ and $e_{1}$ as the bodies evolve inside the commensurability. A comparison with Figure 1 shows that the system follows closely the family of stable ACR for this mass ratio. In other words, the families of ACR that were shown in Section 2 not only give the different equilibrium solutions as function of the angular momentum $\mathcal{L}$, but also correspond to evolutionary tracks within the resonance. Thus, catalogs of ACR such as the one depicted in Figure 1 may not only show us the present location of resonant planetary systems, but also how they evolved (see also Zhou et al. 2008).

As long as the migration is sufficiently slow (adiabatic limit), the evolution of the system within the resonance will not depend on the particular values of $\tau_{a}$ and $\tau_{e}$, but only on the ratio

$$
K=\frac{\tau_{a}}{\tau_{e}}
$$

(see Lee and Peale 2002). In the particular case of Figure 3, the drag parameters were chosen such that $K=0$. Of course different values of $\tau_{a}$ will imply faster or slower orbital decay, but the only effect in the plots will be a modification of the timescale. In particular, the evolutionary track in the $\left(e_{1}, e_{2}\right)$ plane will suffer no change. The adiabatic limit depends on the planetary masses as well as on the resonance (Beaugé et al. 2006). Typically, the smaller the mass ratio, the slower must the migration be to guarantee adiabaticity. If this condition is not satisfied, resonance lock may still occur, 


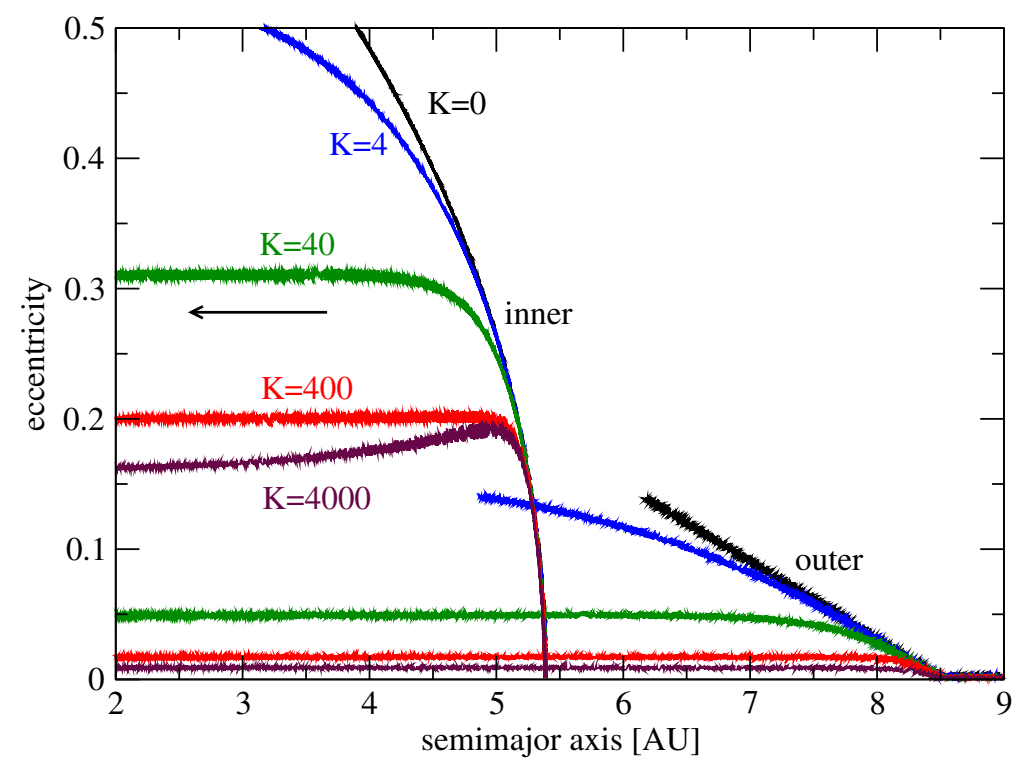

Figure 4. Simulations of planetary migration and capture in the $2 / 1 \mathrm{MMR}$ for fictitious planets with mass ratios similar to the GJ876 resonant planets. The plot shows the evolutionary curves of the eccentricities as function of the decreasing semimajor axes (arrow indicates direction of evolution). Each line corresponds to a different simulation with varied value of $K$. Note that for $K \geqslant 10$ the eccentricities reach equilibrium values.

but the orbital evolution within the commensurability will follow a different path from the families of ACR and, typically, with a much larger amplitude of libration.

The simulation shown in Figure 3 continues until the eccentricity of the inner planet reaches values close to unity and the system is disrupted. This occurs when the semimajor axes are still well above 1 AU. However, a different result is obtained if the migration model includes a significant damping in the eccentricity (i.e. finite values of $\tau_{e}$ and $K>0$ ). Figure 4 shows the results of a series of simulations with $m_{2} / m_{1}=3.15$, similar to the latest edge-on fit for the resonant planets of GJ876 (Laughlin et al. 2005). The plot shows the evolution of the eccentricities as a function of the decaying semimajor axes. Thus, in the graph the system evolves from right to left. Initial conditions were the same as in the previous figure; curves starting at $a=9.5$ AU correspond to the outer planet, while the other branches mark the orbit of the inner body. Each line presents an integration with different value of $K$. For $K=0$ (no eccentricity damping) the eccentricity of the inner planet is quickly excited, reaching almost parabolic orbits after an infall of less than 2 AU. Larger values of $K$ imply smaller eccentricities at a given semimajor axis until, for $K \geqslant 10$, the eccentricities reach an equilibrium value that is constant with respect to the semimajor axis. Note that for $K=4000$ there appears to be a transient interval where $e_{1}$ decreases before reaching a plateau.

The existence of these equilibrium eccentricities alllow the system to suffer large scale migrations while maintaining orbital stability. More importantly, if the planets were initially formed far from the star and captured from quasi-circular orbits, there is only one value of $K$ compatible with the present configuration. In this way, the present eccentricities of the resonant planets could yield information about the characteristics of the gaseous nebula at the final stages of planetary formation. However, this is not an easy task. For example, the current eccentricities of the GJ876 resonant planets seem to indicate that the migration followed a value of $K \sim 40$, while hydrodynamical simulations 
usually give values of $K$ close to unity (Kley et al. 2005). This discrepancy could be evidence in favor of an inner disk in the system (Sandor et al. 2007) or a timely dispersal of the nebula before the eccentricities were excited beyond their present values. A similar study was undertaken for the HD72536 system by Sandor et al. (2007), where the authors discuss the ejection of a hypothetical third planet to account for the large amplitude of libration around the stable ACR.

\section{Orbital Determination of Resonant Planets}

Orbital determination of exoplanets from radial velocity data is a complex process, especially in the case of systems with two planets in mean-motion resonances. The orbital commensurability can give origin to a noticeable periodicity in the radial velocity curve, complicating the separation of both components from the signal. Moreover, two of the most important orbital elements for dynamical studies, the eccentricities $e$ and longitudes of pericenter $\varpi$ are also the most difficult to estimate, since they are given by asymmetries in the quasi-periodic signal. Then, if the individual signals of two planets are mutually affected by their resonant configuration, the precision of the estimation of both $e$ and $\varpi$ can be seriously impaired.

The question then is whether our current knowledge of the planetary parameters are sufficiently reliable to perform detailed dynamical studies, particularly those in which precise values of the orbits are required to reconstruct characteristics of the migration process. Some resonant systems appear well constrained, for example GJ876. Although the orbital elements (in particular the eccentricities) have changed somewhat in the last few years due to an increasing observational database, the overall dynamics of the resonant planets has remained almost invariant. All orbital fits are consistent with a lowamplitude $(0,0)$-ACR. Other resonant systems offer a more complex scenario. A good example of an ill-defined system is HD82943. At present there are two known planets in this system, with orbits consistent with a $2 / 1$ MMR. Both were discovered by the Geneva group (see Ji et al. 2003 for an initial orbital solution), and at present the total number of observations is $N=165$. Of these, 142 were obtained from CORALIE (Mayor et al. 2004), while the remaining 23 come from Keck (Lee et al. 2006). The orbital fits calculated with the complete data set show significant differences with respect to the solution with CORALIE data alone, although in both cases the best fits lead to unstable configurations (Ferraz-Mello et al. 2005a, Lee et al. 2006).

Since it is expected that the real planets should lie in stable regions of the phase space, both Ferraz-Mello et al. (2005a) and Lee et al. (2006) searched for nearby stable orbits with similar weighted r.m.s of the residuals (wrms). All solutions were found to be consistent with resonant motion, usually corresponding to a large-amplitude $(0,0)$-type ACR, although some individual solutions with a circulation of $\Delta \varpi$ were also found. More recently, Goździewski and Konacki (2006) showed that two planets in stable co-orbital motion and non-coplanar orbits could yield similar radial velocity curves, albeit with significantly larger residuals. However, the existence of co-orbital giant planets is far from being established, and there is evidence that planetary bodies with masses larger than $\sim 0.7 M_{\oplus}$ could not be accreted in the equilateral Lagrange points of giant exoplanets (Beaugé et al. 2007). Even so, it is very curious how co-orbital bodies can mimic the radial velocity curve of two bodies in a $2 / 1$ MMR.

Dynamically unstable best fits for resonant planetary systems are not uncommon (e.g. HD73526, HD128311, HD202206), and do not seem very surprising considering the existence of large regions of chaotic motion surrounding the stable resonant regions. However, independent of the dynamical stability, we are also interested in the statistical stability of the orbital solution. In other words, how far can we trust a given best fit, even if 

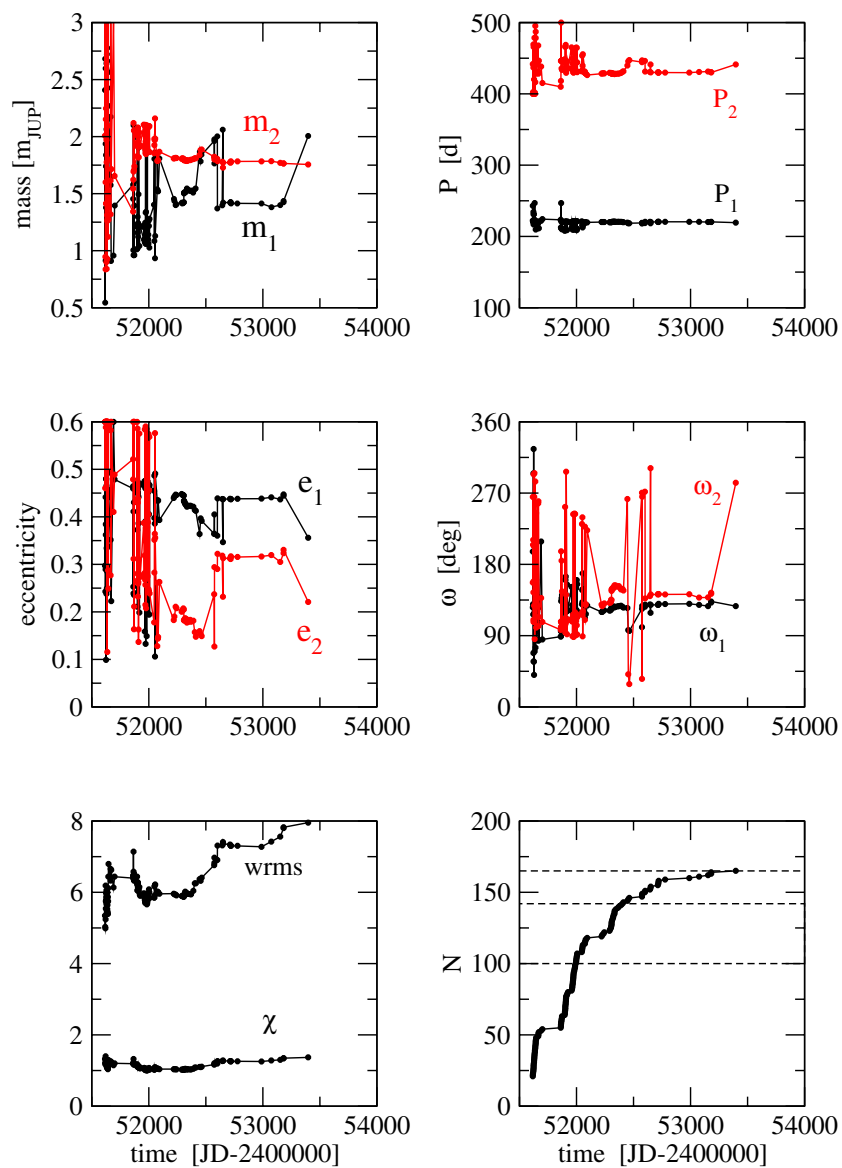

Figure 5. Variation of the parameters of the best multi-Keplerian fit for partial sets of observations, as functions of the times corresponding to the last observation in the set. Data corresponds to complete CORALIE-Keck set.

it leads to stable motion? One possible way is to study the variations of the best fits as function of the number of observations (Beaugé et al. 2008). If the current solution is robust, then we should expect only small and smooth changes in the parameters as a function of the data set number. More importantly, we can then expect that future incorporation of additional observations will not significantly change our knowledge of the system.

Results for multi-Keplerian fits of HD82943 are shown in Figure 5. The lower lefthand frame shows the change in wrms and $\sqrt{\chi^{2}}$. Notice the increase in the value of wrms, particularly over the last few tens of observations. Thus, more data points does not necessarily imply better orbital fits. The lower right-hand plot shows the number of data points as a function of time. The "times" corresponding to the three published orbital fits are identified by horizontal broken lines. With this we can identify in each of the other frames the values of the parameters for each time interval.

Perhaps the most important conclusion from this figure is an apparent non-convergence of the parameters towards defined values. Thus, there is no confidence that future observations will not change the masses and orbital elements of the planets once again, and the current orbital fit of the HD82943 planets does not seem to be reliable. For instance, the passage from $N=164$ to $N=165$ causes very drastic changes in some elements, 

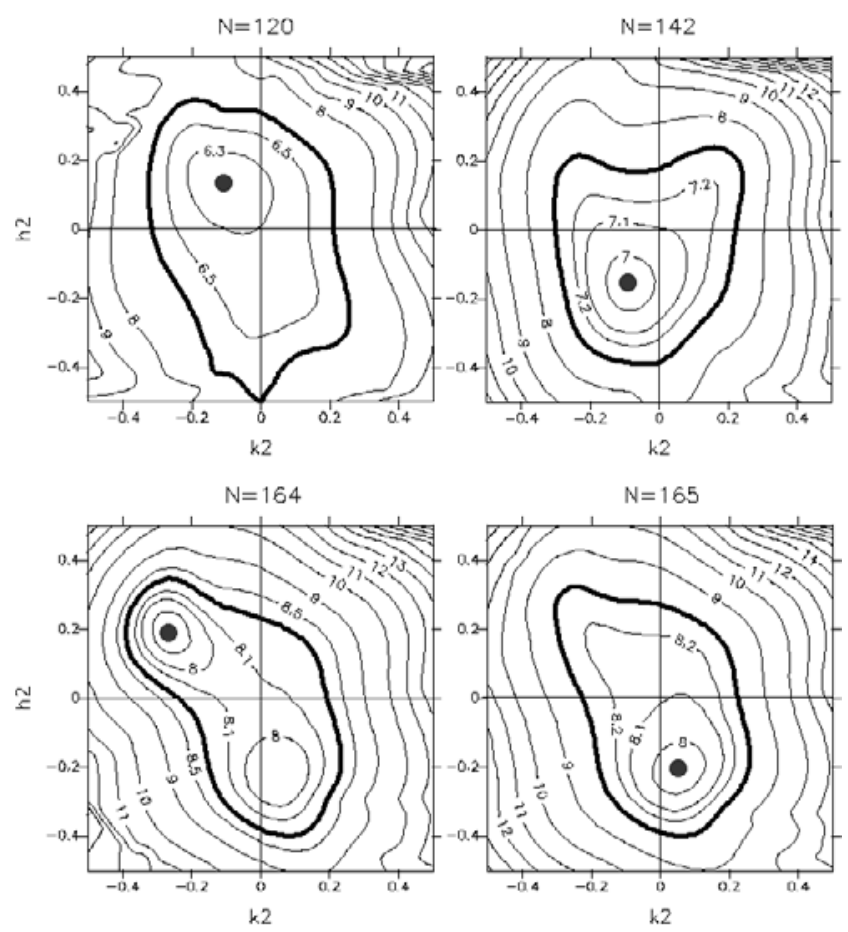

Figure 6. Level curves of constant wrms for four multi-Keplerian orbital fits considering fixed values of $\left(k_{2}, h_{2}\right)=\left(e_{2} \cos \omega_{2}, e_{2} \sin \omega_{2}\right)$ in a regular grid. Each plot was drawn using a different number of data points $N$, simulating the information available at the moment of the publication of each orbital solution. Global minimum is identified with large bullet. Broad level curves represent the 1- $\sigma$ confidence level.

although practically no change in the wrms of the fit (see lower-left hand frame). This seems to imply that both solutions, although very different, are equally consistent with the observational data.

The degree of the statistical instability in the orbital fits is so noticeable that the removal of a single observation can lead to completely different values in some of the orbital parameters. To analyze this sensitivity more globally, we performed a series of multi-Keplerian orbital fits over a grid in the variables $\left(k_{2}, h_{2}\right)=\left(e_{2} \cos \omega_{2}, e_{2} \sin \omega_{2}\right)$. For each point the numerical values of these parameters were fixed, and the fit was done only on the remaining parameters. These were allowed to vary with no restriction, and the resulting wrms corresponds to the best fit for those values of $\left(k_{2}, h_{2}\right)$. We then plotted level curves of wrms in this grid, which give information on the shape and relative depth of that fitness function in the plane.

This procedure was applied to four sets of observations, corresponding to $N=120$, $N=142, N=164$ and $N=165$. Results are shown in Figure 6 . In each case, the global minimum wrms min $_{\text {in }}$ identified by a large bullet. Although close to the minimum the level curves are approximately elliptical, distorsions appear for larger values, indicating that confidence levels obtained from analysis of the correlation matrix are not valid beyond the immediate vicinity of wrms $_{\min }$. The broad level curve in each frame is the $1-\sigma$ confidence level (see Beaugé et al. 2008).

For $N=164$ the figure shows two local minima with almost the same wrms. The global minimum is located in $\omega \sim 140$ degrees, while the other solution ( $\omega \sim 270$ degrees) lies near the global minimum obtained for $N=165$. Thus, not only is the fitness function 
very shallow around the best-fit solution, but sometimes two local minimums can be observed, and very small changes in the data set can lead to one (or the other) being identified as the best fit. Although the individual best fits do change a lot, the shape and extension of the 1- $\sigma$ confidence region appears more robust. Even so, many different types of dynamical configurations coexist within these regions. Thus, although at present it is not possible to choose between them, it seems possible that the HD82943 system lies somewhere inside this region.

A possible origin for this sensitivity to the data set is that HD82943 contains an additional planet. This hypothesis was initially proposed by Goździewski and Konacki (2006) and more recently by Beaugé et al. (2008). The periodograms of the residuals after a two-planet fit and the level of wrms seem to indicate that an additional planet with an orbital period of approximately 900 days could be present.

\section{Conclusions}

Studies of mean-motion resonances (MMR) in exoplanets are far from being purely academic. Several known planetary systems (e.g. GJ876, HD82943, HD73526, HD128311) are believed to lie deep inside the $2 / 1 \mathrm{MMR}$, and other systems may inhabit other commensurabilities, such as the planets 55Cnc-b and 55Cnc-c in the $3 / 1$ or the HD202206 system in the 5/1 MMR. Consequently, a detailed knowledge of the structure of each resonance can yield important insights towards understanding possible locations of real extrasolar bodies.

The large proportion of resonance relations seems to be related to two distinct characteristics. First, MMR are natural nesting places of planets undergoing convergent migration, for example, due to disk-planet interactions. Second, resonances act as a protective mechanism, generating configurations of stable motion in regions of the phase space dominated by instability and close encounters. Thus, MMR constitute privileged locations where exoplanets could be found with large eccentricities and nearby orbits.

However, the dynamical evolution of resonant systems in very sensitive to the initial conditions, and even small uncertainties in orbital fits (as obtained from radial velocity data) may lead to large uncertainties in the evolution of a given multi-planet system. Although some resonant systems are well known (such as GJ876) and their dynamics well mapped, others are less certain. Possibly the most volatile example is HD82943, where even the substraction of a single RV data point can yield completely different orbital solutions and dynamics.

Compared to dynamical studies of our Solar System, where masses and initial conditions are well known, extrasolar planetary systems constitute completely different problems, requiring new methods and approaches. In some ways, resonant exoplanetary systems can be treated as dynamical systems whose initial conditions are not masses or orbital elements, but radial velocity data. It is therefore not surprising that dynamical studies of these systems have become increasingly tied to the process of orbital determination.

\section{Acknowledgments}

This work has been supported by the Argentinean Research Council -CONICET, the Brazilian National Research Council -CNPq, and the São Paulo State Science Foundation -FAPESP. The authors also gratefully acknowledge the support of the CAPES/Secyt programme for scientific collaboration between Argentina and Brazil.

\section{References}

Beaugé, C. \& Michtchenko, T. A. 2003, MNRAS, 341, 760.

Beaugé, C., Ferraz-Mello, S., \& Michtchenko, T. A. 2003, ApJ, 593, 1124. 
Beaugé, C., Michtchenko, T. A., \& Ferraz-Mello, S. 2006, MNRAS, 365, 1160.

Beaugé, C., Sándor, Zs., Érdi, B., \& Süli, À. 2007, AA, 463, 359.

Beaugé, C., Giuppone, C. A., Ferraz-Mello, S., \& Michtchenko, T.A. 2008, MNRAS, submitted.

Ferraz-Mello, S., Beaugé, C., \& Michtchenko, T. A. 2003, CeMDA, 87, 99.

Ferraz-Mello, S., Michtchenko, T. A., \& Beaugé, C. 2005a, ApJ, 621, 473.

Ferraz-Mello, S., Michtchenko, T. A., Beaugé, C., \& Callegari Jr., N. 2005, Lect. Notes Phys., $683,219$.

Fischer, D. A., Marcy, G. W., Butler, R. P., Vogt, S. S., Laughlin, G., Henry, G. W., Abouav, D., Peek, K. M.G., Wright, J. T., Johnson, J. A., McCarthy, C., \& Isaacson, H. 2007, submitted.

Ford, E. B., Lystad, V., \& Rasio, F. A. 2005, Nature, 434, 873.

Goldreich, P. 1965, MNRAS 130, 159.

Goldreich, P. \& Sari, R. 2003, ApJ, 585, 1024.

Goździewski, K. \& Konacki, M. 2006, ApJ, 647, 473.

Ji, J., Liu, L., Kinoshita, H., Zhou, J., Nakai, H., \& Li, G. 2003, ApJ, 591, L57.

Kley, W., Lee, M. H., Murray, N., \& Peale, S. J. 2005, AA, 437, 727.

Laughlin, G. \& Chambers, J. E. 2001, ApJ, 551, L109.

Laughlin, G., Butler, R. P., Fischer, D. A., Marcy, G. W., Vogt, S. S., \& Wolf, A. S. 2005, ApJ, $622,1182$.

Lee, M. H. \& Peale, S. J. 2002, ApJ, 567, 596.

Lee, M. H. 2004, ApJ, 611, 517.

Lee, M. H., Butler, R. P., Fischer, D. A., Marcy, G. W., \& Vogt, S. S. 2006, ApJ, 641, 1178.

Mayor, M., Udry, S., Naef, D., Pepe, F., Queloz, D., Santos, N. C., \& Burnet, M. 2004, AA, $415,391$.

Michtchenko, T. A. \& Ferraz-Mello, S. 2001, AJ, 122, 474.

Michtchenko, T. A. \& Malhotra, R. 2004, Icarus, 168, 237.

Michtchenko, T. A., Beaugé, C., \& Ferraz-Mello, S. 2006, CeMDA, 94, 411.

Michtchenko, T. A., Beaugé, C., \& Ferraz-Mello, S. 2008, in preparation.

Murray, C. D. \& Dermott, S. F. 1999, Solar System Dynamics, Cambridge University Press.

Namouni, F. \& Zhou, J. L. 2006, CeMDA, 95, 245.

Roy, A. E. \& Ovenden, M. W. 1954, MNRAS, 115, 296.

Sándor, Z., Kley, W., \& Klagyivik, P. 2007, AA, 472, 981.

Ward, W. R. 1997, ApJ, 482, L211.

Zhou, L.-Y, Ferraz-Mello, S., \& Sun, Y.-S. 2008, in: Y. -S. Sun, S. Ferraz-Mello, \& J.-L. Zhou, (eds.), Exoplanets: Detection, Formation and Dynamics, Proc. IAU Symposium No. 249 (Suzhou,China), p. 485. 